\title{
Spatial Geometry and Special Relativity: a comparative approach
}

\author{
Geometria Espacial e Relatividade Especial: uma abordagem comparativa \\ Fabiana Botelho Kneubil ${ }^{* 1,2}$ \\ ${ }^{1}$ Department of Science Education, University of Copenhagen, Copenhagen, Denmark \\ ${ }^{2}$ Department of Physics, Federal University of Santa Catarina, Florianópolis, Brazil
}

Recebido em 10 de junho de 2016. Aceito em 20 de julho de 2016

\begin{abstract}
In this work, it is shown the interplay of relative and absolute entities, which are present in both spatial geometry and special relativity. In order to strengthen the understanding of special relativity, we discuss firstly an instance of geometry and the existence of both frame-dependent and frame-independent entities. We depart from a subject well known by students, which is the three-dimensional geometric space in order to compare, afterwards, with the treatment of four-dimensional space in the special relativity. The differences and similarities between these two subjects are also presented in a explicit way, with the goal of improving the comprehension of newcomers on the theory of relativity.
\end{abstract}

Keywords: geometry, special relativity, four-dimensional space, scalar product, relativistic invariant

Nesse trabalho é mostrada a inter-relação das grandezas relativas e absolutas, que estão presentes tanto na geometria espacial quando na relatividade especial. A fim de reforçar o entendimento da relatividade especial, discutimos primeiramente um exemplo da geometria e a existência de entidades dependentes e independentes do referencial. Nós partimos de um assunto bem conhecido dos estudantes, que é o espaço geométrico tridimensional para comparar, depois, com o tratamento do espaço quadridimensional na relatividade especial. As diferenças e similaridades entre esses dois assuntos são também apresentadas de uma maneira explícita, com o objetivo de melhorar a compreensão dos alunos iniciantes na teoria da relatividade.

Palavras-chave: geometria, relatividade especial, espaço quadridimensional, produto escalar, invariante relativístico

\section{Introduction}

The theory of relativity was proposed in 1905 by Einstein and its world view is quite different from the Newtonian mechanics. The uncontrolled popularization of the relativity generated many preposterous views about its content. Maybe, the more improper of them would be the assumption that the relativity shows that 'everything is relative'. On the contrary, the main idea of theory is to determine what is relative in the material world, in order to have a better understanding of what is not. The absolute aspects of the physical universe are, in fact, the major target

*Endereço de correspondência: fkneubil@gmail.com of Einstein's relativity. The special relativity deals with the observation of physical phenomena made in different reference frames, in relative uniform motion. The theory relates the descriptions made of these phenomena and aims at finding absolute laws underlying different descriptions. An important feature is that there is no privileged frame, which allows one to say that the idea underlying on the importance of the reference frame is the desire of describing the same physics in different frames [1]. In this way, the theory distinguishes and correlates two different realms. One of them is related with frame-dependent observations whereas the other, regards absolute quantities which cannot be directly reached by either measures or experiments. The idea 
of relativity gives rise a split of the world into two parts, which are what really exists in the nature and what can be observed. This dichotomy is the essence of relativity since there are both relative and absolute entities in the nature. This was quite emphasized by Minkowski, when he states that

since the postulate comes to mean that only the four-dimensional world in space and time is given by phenomena, but that the projection in space and time may still be undertaken with a certain degree of freedom, I prefer to call it the postulate of the absolute world (or briefly, the world-postulate)( [1], p.83).

This dichotomy is present in physics, mathematics, language and, especially, in our daily life. It is a kind of game based on the distinction between an object and the perceptions of object. For example, if one takes a concrete object, like a cube, there are many ways of knowing what a cube is. This knowledge can begin with the sensorial contact with the physical cube, such as dices or boxes. Doing it, a child experiments the cube with the tough, the eyes, the mouth and so on. After the cube is manipulated we may say that somehow we know or learn about the cube.

This learning on the cube occurs in an abstract way in our mind, since after this experience we recognize all the infinite cubes that exist in the world. There is a pattern which allows one to identify different objects and categorize them as 'cube'. The idea acquired of cube encompasses particular cubes. With this idea one is able to recognize all cubes around us. Therefore, the idea of abstract cube is an absolute and lives in our mind, without mediations. On the other hand, the representations of cube are different and can be done, for example, by means of words, which are relative representations, since they depend on the local, language and culture. Thus, the same idea of cube may be represented by different words, namely würfel, in german, küp, in turkish, or even more complex symbols, as in chinese and japonese languages [2]. We can also represent the cube with figures, as those in fig.(1).

One notes that it is not possible to draw the whole cube. The partiality of representations necessarily incorporates a perspective, which is always particular to an observer.

In both geometric and special relativities, the description of physical phenomena depends on the observer, which is in a given frame. An observer is not able to see directly the whole cube. However, he can see separately each face and reconstruct the whole cube in his mind. The idea of cube and the relationship between their vertices are frame-independent. Hence, there is an absolute entity underlying the relative descriptions. The notion of absolute has always been present in physics, even in geometric objects. In order to understand the relativity applied to the geometry, we discuss in the sequence how the geometric description of the cube is.

\section{The geometric cube}

Take as example the eight vertices of a cube, seen by three different observers. A geometric theory of relativity is based on an idea that each observer describes the same point in a different way. However, these different descriptions keep well-defined relationships between the points. In analytical geometry, a frame is the $x y z$ system. In this paper one uses names of people for designating the frames [2], for example, Mary's frame, denoted by $S^{M}$ and shown in the figure 2(a). For a cube with edge $L$, in $S^{M}$, we have the following coordinates of vertices

$$
\begin{array}{ll}
P_{1}^{M}:(0,0,0) & P_{2}^{M}:(L, 0,0) \\
P_{3}^{M}:(0, L, 0) & P_{4}^{M}:(0,0, L) \\
P_{5}^{M}:(L, L, 0) & P_{6}^{M}:(L, 0, L) \\
P_{7}^{M}:(0, L, L) & P_{8}^{M}:(L, L, L) .
\end{array}
$$
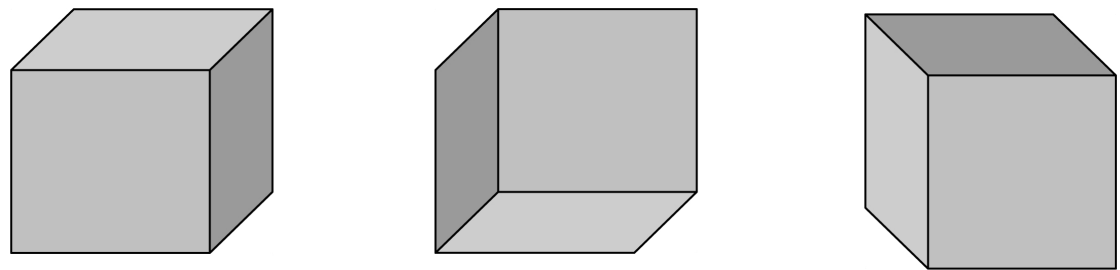

Figure 1: Different images of cube 


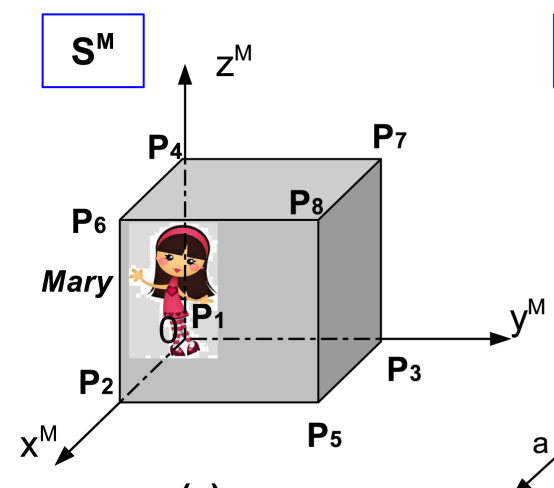

(a)

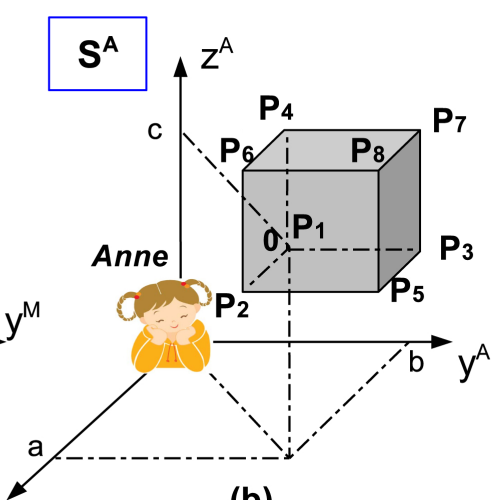

(b)

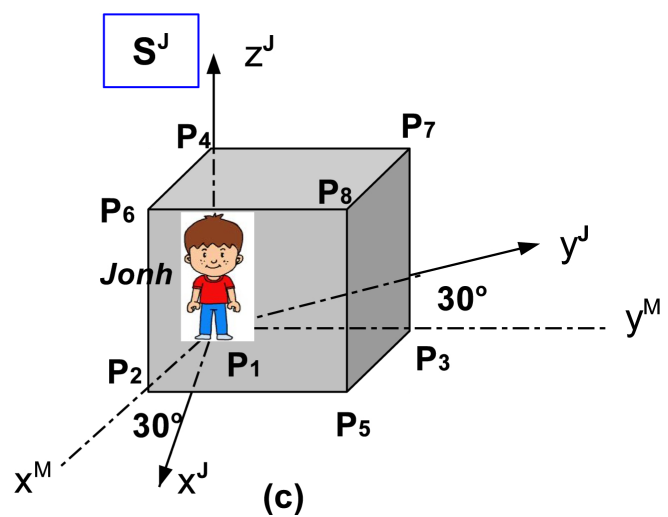

(c)

Figure 2: Cube in different frames

In other frame, such as Anne's frame $S^{A}$, shown in the figure 2 (b), the coordinates of the same vertices are

$$
\begin{aligned}
& P_{1}^{A}:(a, b, c) \\
& P_{2}^{A}:(a+L, b, c) \\
& P_{3}^{A}:(a, b+L, c) \\
& P_{4}^{A}:(a, b, c+L) \\
& P_{5}^{A}:(a+L, b+L, c) \\
& P_{6}^{A}:(a+L, b, c+L) \\
& P_{7}^{A}:(a, b+L, c+L) \\
& P_{8}^{A}:(a+L, b+L, c+L) .
\end{aligned}
$$

One can note that $S^{A}$ is displaced by an arbitrary distance $d$ in relation to $S^{M}$, which can be written as a vector $\vec{d}=(-a,-b,-c)$. Taking another one, the John's frame $S^{J}$, shown in the figure 2(c), the $x$ and $y$ axes are now rotated by $30^{\circ}$ in relation to $S^{M}$ and the descriptions for the same points are

$$
\begin{aligned}
& P_{1}^{J}:(0,0,0) \\
& P_{2}^{J}:\left(\frac{L \sqrt{3}}{2},-\frac{L}{2}, 0\right) \\
& P_{3}^{J}:\left(\frac{L}{2}, \frac{L \sqrt{3}}{2}, 0\right) \\
& P_{4}^{J}:(0,0, L) ; \\
& P_{5}^{J}:\left(\frac{L(\sqrt{3}+1)}{2}, \frac{L(\sqrt{3}-1)}{2}, 0\right)
\end{aligned}
$$

$$
\begin{aligned}
& P_{6}^{J}:\left(\frac{L \sqrt{3}}{2},-\frac{L}{2}, L\right) \\
& P_{7}^{J}:\left(\frac{L}{2}, \frac{L \sqrt{3}}{2}, L\right) \\
& P_{8}^{J}:\left(\frac{L(\sqrt{3}+1)}{2}, \frac{L(\sqrt{3}-1)}{2}, L\right) .
\end{aligned}
$$

The aim of these examples is to verify the dialectical relation between the object and the perceptions of object. The cube is the same for the three observers, whereas its description is not. One could also imagine if we were born in a world without concrete cubes, such as small boxes, dice, and so on. A world in which the cubes can only be known by means of their analytical descriptions, such as the sets of points (1), (2) and (3). It would be hard to reach the concept of cube in this world having only these descriptions. However, there are stable and well established features of the cube, which are common to all particular descriptions. A theory which is able to distinguish relative from absolute entities front of a description is called 'theory of relativity'.

\section{The geometric invariants}

For maintaining the idea of cube, the relations between the vertices should have the same measure in all frames and one says that the distance between two vertices is an invariant, such as the length of the diagonal. For example, the relation between the vertices 7 and 2 in the three frames are given by 


$$
\begin{aligned}
P_{7}^{M}-P_{2}^{M} & =(-L, L, L) \\
P_{7}^{A}-P_{2}^{A} & =(-L, L, L) \\
P_{7}^{J}-P_{2}^{J} & =\left(\frac{L}{2}-\frac{L \sqrt{3}}{2}, \frac{L \sqrt{3}}{2}+\frac{L}{2}, L\right) .
\end{aligned}
$$

One notes that these results are not equal to three frames, however, if we consider that each substraction is a generic vector $\vec{A}$, the length of diagonal is given by the modulus of the vector $|\vec{A}|=\sqrt{x^{2}+y^{2}+z^{2}}$. Thus, in $S^{M}, S^{A}$ and $S^{J}$, such vectors are $\vec{A}^{M}=(-L, L, L), \vec{A}^{A}=(-L, L, L)$ and $\vec{A}^{J}=\left(\frac{L}{2}-\frac{L \sqrt{3}}{2}, \frac{L \sqrt{3}}{2}+\frac{L}{2}, L\right)$, and their modulus are

$$
\begin{aligned}
& \sqrt{\left(P_{7}^{M}-P_{2}^{M}\right)^{2}}=\sqrt{\left(P_{7}^{A}-P_{2}^{A}\right)^{2}} \\
& =\sqrt{\left(P_{7}^{J}-P_{2}^{J}\right)^{2}}=L \sqrt{3} .
\end{aligned}
$$

The geometric meaning of this result is simple: $L \sqrt{3}$ is the distance between vertices 7 and 2 , which is an invariant. In the two first cases, this invariant is originated by translating frames $\left(S^{M}\right.$ and $\left.S^{A}\right)$, whereas the third, the invariant comes by rotating frame (30-degree of the axes $x$ and $y$ in $S^{J}$ ). The invariants coming by rotating frames are quite relevant in geometric relativity. For example, the single point $\mathrm{P}$ in figure 3(a), is written as

$$
\vec{A}=r \cos \theta \hat{i}+r \operatorname{sen} \theta \hat{j}+0 \hat{k}
$$

whereas in $x^{\prime} y^{\prime} z^{\prime}$ system, which is rotated by a angle $\alpha$ in relation to $x y$ axes, the new description of $P$, shown in the figure $3(b)$, is

$$
\vec{A}^{\prime}=r \cos \varphi \hat{i}+r \operatorname{sen} \varphi \hat{j}+0 \hat{k}
$$

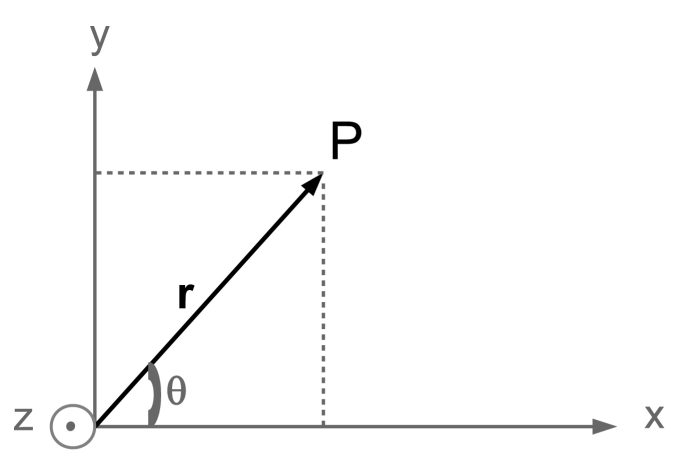

(a) where $\varphi=\theta-\alpha$.

Although the both descriptions are different, one shows that the modulus of $\vec{A}$ is an invariant, calculating $|\vec{A}|=\sqrt{x^{2}+y^{2}+z^{2}}$. In $x y z$ system we have

$$
\begin{aligned}
& |\vec{A}|=\sqrt{r^{2} \cos ^{2} \theta+r^{2} \operatorname{sen}^{2} \theta} \\
& =\sqrt{r^{2}\left(\cos ^{2} \theta+\operatorname{sen}^{2} \theta\right)}=\sqrt{r^{2}}=r,
\end{aligned}
$$

and in the $x^{\prime} y^{\prime} z^{\prime}$ system, the same operation is

$$
\begin{aligned}
& |\vec{A}|=\sqrt{r^{2} \cos ^{2} \varphi+r^{2} \operatorname{sen}^{2} \varphi} \\
& =\sqrt{r^{2}\left(\cos ^{2} \varphi+\operatorname{sen}^{2} \varphi\right)}=\sqrt{r^{2}}=r .
\end{aligned}
$$

These results show that the modulus of a vector does not depend on neither the reference system nor the angle rotated. A theory of relativity is one which transforms the coordinates from a frame into another one, i.e., it transforms $(x, y, z)$ of $P$ into $\left(x^{\prime}, y^{\prime}, z^{\prime}\right)$. We note that the vector $\vec{A}$ does not depend on the $\alpha$ in two frames and, hence, we have to related $\alpha$ with $\theta$ and $\varphi$, by means of the following trigonometric relations.

$$
\begin{aligned}
& \cos \varphi=\cos (\theta-\alpha)=\cos \theta \cos \alpha+\operatorname{sen} \theta \operatorname{sen} \alpha \\
& \operatorname{sen} \varphi=\operatorname{sen}(\theta-\alpha)=\operatorname{sen} \theta \cos \alpha-\operatorname{sen} \alpha \cos \theta
\end{aligned}
$$

Therefore, the equations which relate $x, y$ and $z$ with $x^{\prime}, y^{\prime}$ and $z^{\prime}$ are

$$
\begin{aligned}
x & =r \cos \theta \\
y & =r \operatorname{sen} \theta \\
z & =0 \\
x^{\prime} & =r[\cos \theta \cos \alpha+\operatorname{sen} \theta \operatorname{sen} \alpha] \\
y^{\prime} & =r[\operatorname{sen} \theta \cos \alpha-\operatorname{sen} \alpha \cos \theta] \\
z^{\prime} & =0 .
\end{aligned}
$$

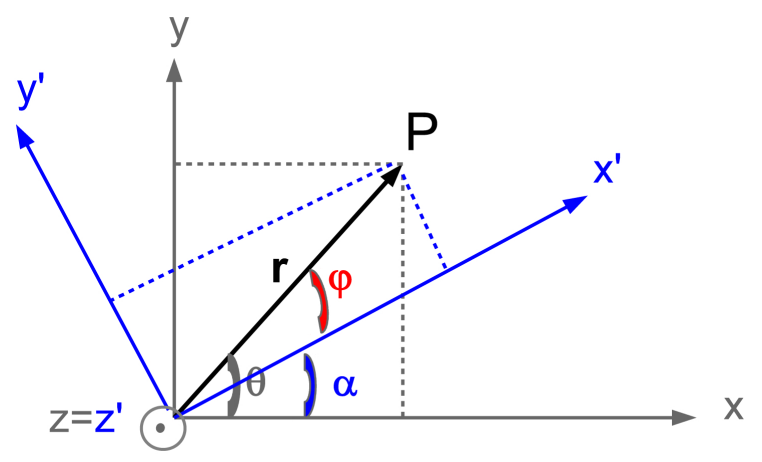

(b)

Figure 3: Description of vector in rotating frames 
In order to obtain a set of equations which transform $x y z \rightarrow x^{\prime} y^{\prime} z^{\prime}$, we replace eqs.(6) in (7), yielding

$$
\begin{aligned}
& x^{\prime}=\cos \alpha x+\operatorname{sen} \alpha y \\
& y^{\prime}=-\operatorname{sen} \alpha x+\cos \alpha y \\
& z^{\prime}=z,
\end{aligned}
$$

which can be also represented by a matricial form

$$
\left[\begin{array}{l}
x^{\prime} \\
y^{\prime} \\
z^{\prime}
\end{array}\right]=\left[\begin{array}{ccc}
\cos \alpha & \operatorname{sen} \alpha & 0 \\
-\operatorname{sen} \alpha & \cos \alpha & 0 \\
0 & 0 & 1
\end{array}\right]\left[\begin{array}{l}
x \\
y \\
z
\end{array}\right] .
$$

These equations are well known from rotating geometry and are quite useful, since they determine the coordinates of a same vector in different frames and allow one to make changes of frame, in a analytical way, without constructing the geometric figure. Therefore, the figure of cube can be abandoned and the calculus is made only with the points. Returning to vertices $P_{7}^{M}$ and $P_{2}^{M}$, described by $(L, 0,0)$ and $(0, L, L)$ in $S^{M}$, the result (8) allows to obtain $P_{2}^{J}$ and $P_{7}^{J}$ in $S^{J}$, which is $\theta$-degree rotated in relation to $S^{M}$. The figure (4) shows these points in $S^{M}$ and their corresponding vectors.

Using the eqs.(8), the same vectors can be written in $S^{J}$ as

$$
\begin{aligned}
& \vec{A}_{2 x}^{\prime}=x \cos \theta+y \operatorname{sen} \theta \\
& =L \cos \theta+0 \operatorname{sen} \theta \\
& \vec{A}_{2 y}^{\prime}=-x \operatorname{sen} \theta+y \cos \theta \\
& =-L \operatorname{sen} \theta+0 \cos \theta \\
& \vec{A}_{2 z}^{\prime}=z=0,
\end{aligned}
$$

which yields $\vec{A}_{2}^{\prime}=(L \cos \theta,-L \operatorname{sen} \theta, 0)$. Similarly, we write the vector which represents the point

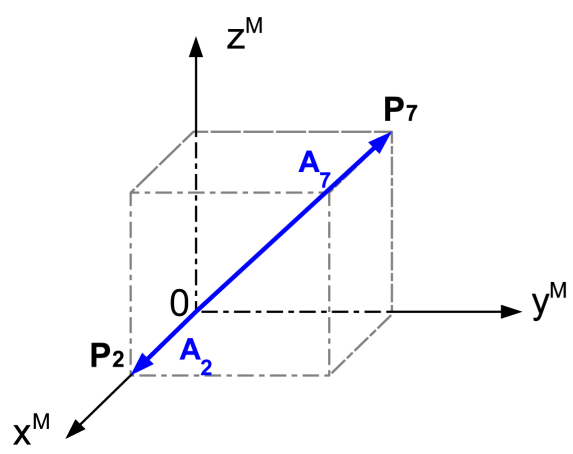

7 in $S^{J}$ as

$$
\begin{aligned}
& \vec{A}_{7 x}^{\prime}=x \cos \theta+y \operatorname{sen} \theta \\
& =0 \cos \theta+L \operatorname{sen} \theta \\
& \vec{A}_{7 y}^{\prime}=-x \operatorname{sen} \theta+y \cos \theta \\
& =0 \operatorname{sen} \theta+L \cos \theta \\
& \vec{A}_{7 z}^{\prime}=z=L
\end{aligned}
$$

which yields $\vec{A}_{7}^{\prime}=(L \operatorname{sen} \theta, L \cos \theta, L)$. It is easy to see that they are different in each frame, but the distance between $P_{7}$ and $P_{2}$ remains unchanged, since it corresponds to the length of the diagonal. Calculating $\vec{A}_{7}-\vec{A}_{2}$ and $\vec{A}_{7}^{\prime}-\vec{A}_{2}^{\prime}$, we have

$$
\begin{aligned}
& \vec{A}_{7}-\vec{A}_{2}=-L \hat{i}+L \hat{j}+L \hat{k}=(-L, L, L) \\
& d_{7-2}=L \sqrt{3} \\
& \vec{A}_{7}^{\prime}-\vec{A}_{2}^{\prime}=L \operatorname{sen} \theta \hat{i} \\
& +L \cos \theta \hat{j}+L \hat{k}-L \cos \theta \hat{i}+L \operatorname{sen} \theta \hat{j}-0 \hat{k} \\
& =L(\operatorname{sen} \theta-\cos \theta) \hat{i}+L(\cos \theta+\operatorname{sen} \theta) \hat{j}+L \hat{k} \\
& d_{7-2}^{\prime}= \\
& \sqrt{L^{2}(\operatorname{sen} \theta-\cos \theta)^{2}+L^{2}(\cos \theta+\operatorname{sen} \theta)^{2}+L^{2}} \\
& d_{7-2}^{\prime}=L \sqrt{3}
\end{aligned}
$$

The main goal of these demonstrations is to emphasize that in spatial geometry there are entities frame-independent. These entities are invariants and are obtained by means of some mathematical operation between two frames. In general, the invariants are hidden behind the descriptions and for obtaining them it is necessary to make an operation with the coordinates. In spatial geometry it is usual to call the theory of coordinate transformations as relativity by rotations. In the Einstein's special relativity there is also a set of equations which transforms

$$
\begin{aligned}
& \vec{A}_{2}=L \hat{\imath}+0 \hat{\jmath}+0 \hat{k} \\
& \vec{A}_{7}=0 \hat{\imath}+L \hat{\jmath}+L \hat{k}
\end{aligned}
$$

Figure 4: Vectors $\vec{A}_{2}$ and $\vec{A}_{7}$ in $S^{M}$ 
descriptions of physical events between two frames. In the following section we show the differences and similarities between spatial relativity and special relativity.

\section{The relativistic cube}

In geometry the game between the object and their representations can be complex and depends on the observers. The Einstein's theory of relativity is similar and its purpose is to find the absolute laws which determine the apparent description of phenomena. The great difference in this theory is that it relates the descriptions of moving observers.

Events are particularly importante in relativity. They are occurrences which can be described by four coordinates in a frame: one instant in time and one point in tridimensional space. Minkowski introduced this concept as a world-point, saying that

the objects of our perception invariably include places and times in combination. Nobody has ever notice a place except at a time, or a time except at a place. [... A point of space at a point of time, that is, a system of values $x, y, z, t$, I will call a world-point. The multiplicity of all thinkable $x, y, z, t$ systems of values we will christen the world ([3], p.76).

The occurrence of an event is something absolute, i.e., if it occurs in a given frame, it will also occur in all the other ones. However, the instant and the position which they occur depend on the frame. The theory is based on the idea that different observers describe the same event in different ways, by means of different coordinates. A frame is a system with three spatial axes added with a time-like coordinate and, differently from spatial geometry, the space is four-dimensional. Therefore, the description of an event is made by means of a point with four coordinates. An event in $S^{M}, S^{A}$ and $S^{J}$ is described as $\left(c t^{M} ; x^{M}, y^{M}, z^{M}\right),\left(c t^{A} ; x^{A}, y^{A}, z^{A}\right)$ and $\left(c t^{J} ; x^{J}, y^{J}, z^{J}\right)$, where the time coordinate were multiplied by $c$ so that all components have the same dimension.

An event in relativity is similar to a vertex of the cube in spatial geometry. In the case of geometry, the idea of cube is defined by a specific relation between the vertices, which is always equal for different frames and, therefore, is absolute. We used the calculus of the diagonal for elucidating the existence of invariants. In special relativity, there are also relationships between events, which preserve and maintain some abstract entity, that is analogous to the idea of cube.

In order to understand this abstract relativistic cube, we consider another example, which in turn, cannot be drawn in the same way, since we are dealing with a four-dimensional space. Moreover, one needs to consider the time coordinate in the vertex of this 'relativistic cube', i.e., the object is constructed by events. Take a ruler with length $L$ and two pens coupled at their ends, one is red and other, blue. The pens can mark a paper with two different colorful dots. This ruler comes down and mark these two points, at the first time, at an instant $t_{1}$. Then, the ruler changes its position and repeats the colorful marks in a different $y$-coordinate, at the instant $t_{2}$. This situation is illustrated in figure (5), where $a$ and $b$ are the distances of the ruler from the origin at the instants $t_{1}$ and $t_{2}$ respectively.

The two descents of the ruler generate four events, i.e., four occurrences in the four-dimensional space, which are described by:

$$
\begin{array}{ll}
E_{1}:\left(c t_{1} ; 0, a, 0\right), \\
E_{2}:\left(c t_{1} ; 0, a+L, 0\right), \\
E_{3}:\left(c t_{2} ; 0, b, 0\right), \\
E_{4}:\left(c t_{2} ; 0, b+L, 0\right) .
\end{array}
$$

One can also situate these four events in a fourdimensional space in the same way that we represented the vertices of geometric cube in a threedimensional space. One notes that the coordinates $x$ and $z$ are always zero, since the ruler is placed along the $y$-axis. This facilitates the construction of these events, which are shown in figure 6 .

These four events seem to be the vertices of a cube, representing together one of its face, however, it is a face of an imaginary cube, since one of the axis is the time and the figure is a parallelogram.

The purpose of this example is to show that the events in relativity are represented by means of four coordinates, i.e., there is one more coordinate than the description of a point in analytical geometry. In both Physics and Mathematics the change of frame is quite important. We showed that the coordinates of vertices of a cube are different for each frame and that it is possible to transform a set of coordinates 

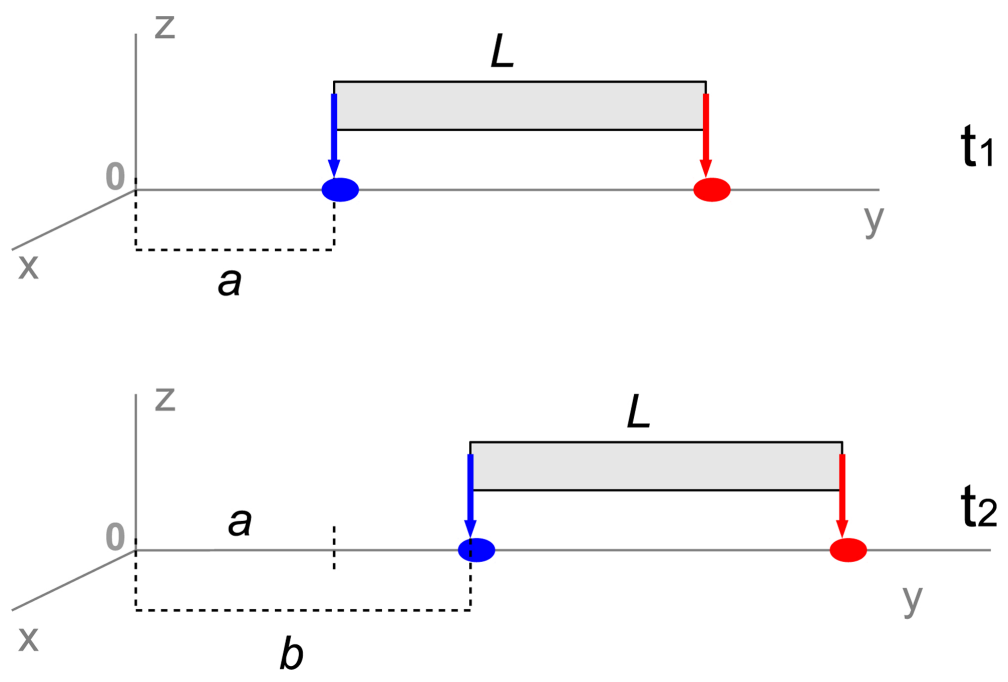

Figure 5: Ruler in different positions

from a frame into another one, by means of the equations (8), which are called relativity by rotations. In special relativity, the set of equations which makes it are the Lorentz transformations.

In relativity, we have four coordinates and, therefore, the Lorentz transformations transform ct; $x y z \rightarrow$ $c t^{\prime} ; x^{\prime} y^{\prime} z^{\prime}$. There are two essential differences between eq.(8) and the Lorentz equations: (i) in relativity there are four equations involving four coordinates and (ii) there is no angle of rotation between frames, but instead it there is a relative velocity between frames. Thus, the angle in eq. 8 ) is substituted by a relative velocity $v$ between two frames. For simplifying the calculation, we adopt the relative velocity $v$ only in the $y$-axis and, therefore, there are no changes in $x$ and $z$ coordinates. Taking $S^{M}$ and $S^{J}$, their descriptions of an event are $\left(c t^{M} ; x^{M}, y^{M}, z^{M}\right)$ and $\left(c t^{J} ; x^{J}, y^{J}, z^{J}\right)$. If John movies relative to Mary with a constant velocity $v$, along the $y$-axis, and they adopt a single space and time origin, the mathematical operations which relate their sets of four coordinates are

$$
\begin{aligned}
x^{J} & =x^{M}, \\
y^{J} & =\gamma\left(y^{M}-v t^{M}\right), \\
z^{J} & =z^{M}, \\
t^{J} & =\gamma\left(t^{M}-\frac{v}{c^{2}} y^{M}\right) .
\end{aligned}
$$

where $\gamma=1 / \sqrt{1-\frac{v^{2}}{c^{2}}}$ is called Lorentz factor 4 . Considering the four events 10 as being in $S^{M}$, one can obtain their description in $S^{J}$, which moves with relative velocity $v$. Calculating $y$ and $t$ coordinates, we have

\section{Event 1}

Mary $-E_{1}:\left(c t_{1} ; 0, a, 0\right) \longrightarrow$

$\overline{\text { John }}-E_{1}:(? ; 0, ?, 0)$

$$
\begin{aligned}
& t^{J}=\gamma\left(t^{M}-\frac{v}{c^{2}} y^{M}\right)=\gamma\left(t_{1}-\frac{v}{c^{2}} a\right) \\
& y^{J}=\gamma\left(y^{M}-v t^{M}\right)=\gamma\left(a-v t_{1}\right) \\
& \underline{\text { John }}-E_{1}:\left(\gamma\left(t_{1}-\frac{v}{c^{2}} a\right) ; 0, \gamma\left(a-v t_{1}\right), 0\right)
\end{aligned}
$$

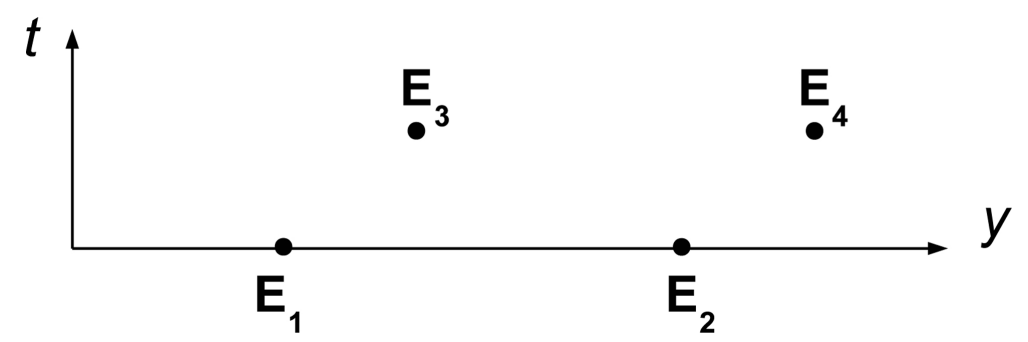

Figure 6: Events - relativistic cube 


\section{Event 2}

Mary $-E_{2}:\left(c t_{1} ; 0, a+L, 0\right) \longrightarrow$

$\overline{\mathrm{John}}-E_{2}:(? ; 0, ?, 0)$

$t^{J}=\gamma\left(t^{M}-\frac{v}{c^{2}} y^{M}\right)=\gamma\left[t_{1}-\frac{v}{c^{2}}(a+L)\right]$

$y^{J}=\gamma\left(y^{M}-v t^{M}\right)=\gamma\left(a+L-v t_{1}\right)$

$\underline{\mathrm{John}}-E_{2}:\left(\gamma\left[t_{1}-\frac{v}{c^{2}}(a+L)\right] ; 0, \gamma(a+L-\right.$ $\left.\left.v t_{1}\right), 0\right)$

\section{Event 3}

$$
\begin{aligned}
& \underline{\text { Mary }}-E_{3}:\left(c t_{2} ; 0, b, 0\right) \longrightarrow \\
& \overline{\mathrm{John}}-E_{3}:(? ; 0, ?, 0) \\
& t^{J}=\gamma\left(t^{M}-\frac{v}{c^{2}} y^{M}\right)=\gamma\left(t_{2}-\frac{v}{c^{2}} b\right) \\
& y^{J}=\gamma\left(y^{M}-v t^{M}\right)=\gamma\left(b-v t_{2}\right) \\
& \underline{\text { John }}-E_{3}:\left(\gamma\left(t_{1}-\frac{v}{c^{2}} b\right) ; 0, \gamma\left(b-v t_{1}\right), 0\right)
\end{aligned}
$$

\section{Event 4}

Mary $-E_{4}:\left(c t_{2} ; 0, b+L, 0\right) \longrightarrow$

$\overline{\mathrm{John}}-E_{4}:(? ; 0, ?, 0)$

$$
\begin{aligned}
& t^{J}=\gamma\left(t^{M}-\frac{v}{c^{2}} y^{M}\right)=\gamma\left[t_{2}-\frac{v}{c^{2}}(b+L)\right] \\
& y^{J}=\gamma\left(y^{M}-v t^{M}\right)=\gamma\left(b+L-v t_{2}\right)
\end{aligned}
$$

$\underline{\text { John }}-E_{4}:\left(\gamma\left[t_{2}-\frac{v}{c^{2}}(b+L)\right] ; 0, \gamma(b+L-\right.$ $\left.\left.v t_{2}\right), 0\right)$

One repares that the descriptions in $S^{J}$ are quite weird and the classical intuition does not reach their meanings, since we are dealing with a fourdimensional space and the spatial and temporal coordinates appear mixed together. On the other hand, underlying all these descriptions must have something common in both frames. This abstract entity is the absolute which does not depend on the changing frame and is an invariant by Lorentz transformations. The relativistic invariant is hidden and is necessary to perform some mathematical operation to find it.

\section{The relativistic invariants}

In the geometric cube, the invariant is found by means of the relation between two vertices and we took as example the diagonal length. The operation to obtain the diagonal length is the modulus of vector $\vec{A}$, which is calculated by $\sqrt{x^{2}+y^{2}+z^{2}}$. In relativity, there is one more coordinate which leads one to think whether the distance between two events is calculated by the same formula, just adding the $t$-coordinate term, as represented in figure (7).

The answer to this question is no and was introduced in relativity by Minkowski [3], who realized that the distinction between space-like and time-like coordinates is made by means of changing the sign. In relativity the definition of the distance between two events $d s^{2}$ is given by

$$
d s^{2}=c^{2} t^{2}-x^{2}-y^{2}-z^{2},
$$

and is called relativistic interval. This strange sum has an important meaning within theory and represents the relativistic invariants, similar to the geometry. In Euclidean space, a vector squared corresponds to the operation $A^{2}=\vec{A} \cdot \vec{A}=A_{x}^{2}+A_{y}^{2}+A_{z}^{2}$, i.e., the dot product of $\vec{A}$ by itself. This result, as we already mentioned, is an invariant, a scalar entity. The great new of relativity is the re-definition of dot product, which yields invariant entities. In a fourdimensional space, the dot product of a 4 -vector $A=\left(A_{0} ; A_{x}, A_{y}, A_{z}\right)$ by itself is

$$
A^{2}=A \cdot A=A_{0}^{2}-A_{x}^{2}-A_{y}^{2}-A_{z}^{2} .
$$

The minus signs in front of the spatial coordinates are essential for the dot product $A^{2}$ to be frameindependent. All scalar products of four-vectors are relativistic invariants, however, only some of them are relevant and have meaning within the theory. The first invariant we present is the four-distance $\sqrt{A^{2}}$, which replaces the typical Euclidean notion

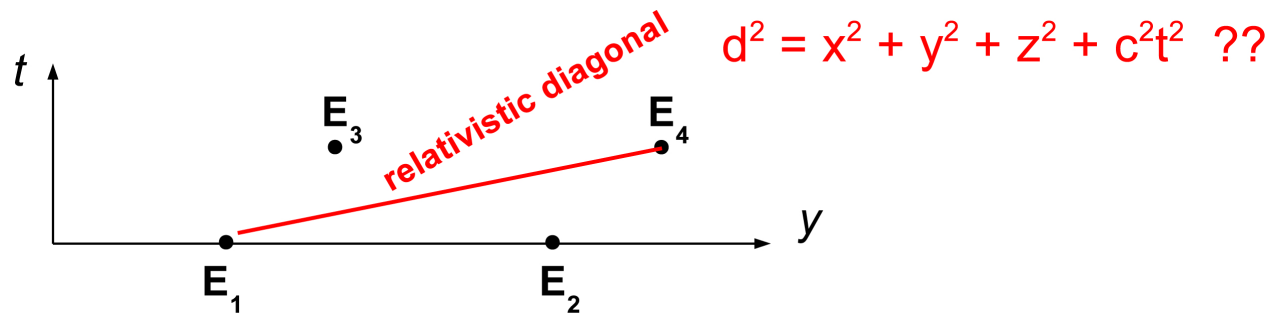

Figure 7: Relativistic diagonal 
of distance [5]. One can show, therefore, that this abstract entity is equal for both $S^{M}$ and $S^{J}$ by calculating the sum (5) in each frame. For $S^{M}$, it is

$$
\left(d s^{M}\right)^{2}=c^{2}\left(t^{M}\right)^{2}-\left(x^{M}\right)^{2}-\left(y^{M}\right)^{2}-\left(z^{M}\right)^{2},
$$

whereas for $S^{J}$, the sum is

$$
\left(d s^{J}\right)^{2}=c^{2}\left(t^{J}\right)^{2}-\left(x^{J}\right)^{2}-\left(y^{J}\right)^{2}-\left(z^{J}\right)^{2} \text {. }
$$

In order to compare these two intervals, we take the events $E_{4}$ and $E_{1}$ of previous example and calculate the interval between them.

$$
E_{1}\left\{\begin{array}{l}
M:\left(t_{1} ; 0, a, 0\right) \\
J:\left[\gamma\left(t_{1}-\frac{v}{c^{2}} a\right) ; 0, \gamma\left(a-v t_{1}\right), 0\right]
\end{array}\right.
$$

$E_{4}\left\{\begin{array}{l}M:\left(t_{2} ; 0, b+L, 0\right) \\ J:\left(\gamma\left[t_{2}-\frac{v}{c^{2}}(b+L)\right] ; 0, \gamma\left(b+L-v t_{2}\right.\right. \\ 0)\end{array}\right.$

Replacing these coordinates in eqs.(14) and (15), the relativistic intervals for $S^{M}$ and $S^{J}$ are presented in the sequence.

Mary : $\left(d s^{M}\right)^{2}=c^{2}\left(t_{2}-t_{1}\right)^{2}-(b+L-a)^{2}$

$\underline{\overline{\text { John }}}:\left(d s^{J}\right)^{2}=c^{2} \gamma^{2}\left[t_{2}-\frac{v}{c^{2}}(b+L)-t_{1}+\frac{v}{c^{2}} a\right]^{2}$ $-\gamma^{2}\left[b+L-v t_{2}-a+v t_{1}\right]^{2}$

$\left(d s^{J}\right)^{2}=c^{2} \gamma^{2}\left[\left(t_{2}-t_{1}\right)-\frac{v}{c^{2}}(b+L-a)\right]^{2}$

$-\gamma^{2}\left[(b+L-a)-v\left(t_{2}-t_{1}\right)\right]^{2}$

$\left(d s^{J}\right)^{2}=\gamma^{2}\left\{\left[c\left(t_{2}-t_{1}\right)-\frac{v}{c}(b+L-a)\right]^{2}\right.$

$\left.-\left[(b+L-a)-v\left(t_{2}-t_{1}\right)\right]^{2}\right\}$,

with the development of the sum of squares, the mixed term is canceled, which yields

$$
\begin{aligned}
& \left(d s^{J}\right)^{2}=\gamma^{2}\left\{c^{2}\left(t_{2}-t_{1}\right)^{2}+\frac{v^{2}}{c^{2}}(b+L-a)^{2}\right. \\
& \left.-(b+L-a)^{2}-v^{2}\left(t_{2}-t_{1}\right)^{2}\right\} \\
& \quad\left(d s^{J}\right)^{2}=\gamma^{2}\left\{\left(c^{2}-v^{2}\right)\left(t_{2}-t_{1}\right)^{2}-(b+L-a)^{2}\right. \\
& \left.\left[1-\frac{v^{2}}{c^{2}}\right]\right\} .
\end{aligned}
$$

Finally, using $\gamma^{2}=\frac{c^{2}}{c^{2}-v^{2}}$, the result is $\left(d s^{J}\right)^{2}=$ $c^{2}\left(t_{2}-t_{1}\right)^{2}-(b+L-a)^{2}$, which corresponds to the same value for $S^{M}$.

\section{Concluding remarks}

In this work, we show differences and similarities between three-dimensional geometry and special relativity. The treatment of physical phenomena in a four-dimensional space requires a new kind of mathematics, since the spatial and temporal coordinates are mixed together. Albeit the mathematical operations are different, the way of thinking is similar. The existent entities in Euclidean geometry have their corresponding analogues in special relativity, ) which are summarized in table (1).

We mentioned in this paper only one relativistic invariant, namely the four-distance. However, there are other important invariants, generated from different dot products. For example, the proper time $\tau$, coming from a specific time-like interval $[5]$ and the mass, which is defined by the squared of the 4 -momentum $p^{2}[6]$. This comparison between geometry and relativity is a starting point for students to plunge in the four-dimensional world of physics. It aims at turning the subject more understandable for students not acquainted with the theory of relativity. On the other hand, both teachers and student have to be aware that the interpretation of fourdimensional entities in relativity is quite complex, since it deals with abstract and absolute concepts. In this sense, there is no continuous path from a lesser to a higher dimension and, hence, the understanding of four-dimensional entities can only be achieved by means of mathematical constructions.

Table 1: Comparative approach - Geometry and Relativity

\begin{tabular}{lcc}
\hline & GEOMETRY & RELATIVITY \\
\hline mathematical space & 3-dimensional & 4-dimensional \\
\hline coordinates & $x, y, z$ & $t ; x, y, z$ \\
\hline cube (points) & vertices & events \\
\hline description of points & by means of a & by means of a \\
& vector $\vec{A}=\left(A_{x}, A_{y}, A_{z}\right)$ & 4-vector $A=\left(A_{t} ; A_{x}, A_{y}, A_{z}\right)$ \\
\hline transformations & 3 angle-dependent equations & 4 velocity-dependent equations \\
& Rotations - eqs.(8) & Lorentz Transform.- eqs. $\sqrt{11}$ \\
\hline scalar product & $A^{2}=x^{2}+y^{2}+z^{2}$ & $A^{2}=c^{2} t^{2}-x^{2}-y^{2}-z^{2}$ \\
\hline meaning of & distance between two vertices & four-distance \\
$\sqrt{A^{2}}$ & & \\
\hline
\end{tabular}




\section{Acknowledgements}

I am very grateful for discussions with M.R.Robilotta on the content of this paper. I also thank the financial support provided by CAPES (Coordenação de Apoio à Pesquisa - Brazilian Agency) (BEX 0816/159).

\section{References}

[1] A. Einstein, H.A. Lorentz, H. Minkowski and H. Weyl, The Principle of Relativity (Dover, New York, 1952).

[2] M.J. Bechara, J.L.M. Duarte, M.R. Robilotta and S. Salém, Física 4, Lecture Notes (Departamento de Física, Universidade de São Paulo, 2014).

[3] H. Minkowski, Space and Time - Minkowski's Papers on Relativity - Free version (Minkowski Institute Press, Montreal, 1909).

[4] D. Griffiths, Introduction to Elementary Particles Second Revised Edition (Wiley-VCH Press, Berlin, 2008).

[5] J. Wheeler and E. Taylor, Spacetime Physics (W.H. Freeman and Company, New York, 1992).

[6] L.B. Okun, Physics Today 42,31 (1989). 